\title{
Autophagy in the posterior interosseous nerve of patients with type 1 and type 2 diabetes mellitus: an ultrastructural study
}

\author{
Ayman A. M. Osman • Lars B. Dahlin • \\ Niels O. B. Thomsen • Simin Mohseni
}

Received: 31 October 2014 / Accepted: 27 November 2014 / Published online: 19 December 2014

(C) Springer-Verlag Berlin Heidelberg 2014

\begin{abstract}
Aims/hypothesis We addressed the question of whether the autophagy pathway occurs in human peripheral nerves and whether this pathway is associated with peripheral neuropathy in diabetes mellitus.

Methods By using electron microscopy, we evaluated the presence of autophagy-related structures and neuropathy in the posterior interosseous nerve of patients who had undergone carpal tunnel release and had type 1 or type 2 diabetes mellitus, and in patients with no diabetes (controls).

Results Autophagy-related ultrastructures were observed in the samples taken from all patients of the three groups. The number of autophagy-associated structures was significantly higher $(p<0.05)$ in the nerves of patients with type 1 than type 2 diabetes. Qualitative and quantitative evaluations of fascicle area, diameter of myelinated and unmyelinated nerve fibres, the density of myelinated and unmyelinated fibres and the gratio of myelinated fibres were performed. We found degeneration and regeneration of a few myelinated axons in controls, and a well-developed neuropathy with the loss of large myelinated axons and the presence of many small ones in patients with diabetes. The pathology in type 1 diabetes was more extensive than in type 2 diabetes.

Conclusions/interpretation The results of this study show that the human peripheral nerves have access to the autophagy
\end{abstract}

A. A. M. Osman $\cdot$ S. Mohseni $(\bowtie)$

Department of Clinical and Experimental Medicine, Division of Cell Biology, Linköping University, SE-581 83 Linköping, Sweden

e-mail: simin.mohseni@liu.se

L. B. Dahlin • N. O. B. Thomsen

Department of Clinical Sciences Malmö - Hand Surgery, Lund

University, Malmö, Sweden

L. B. Dahlin • N. O. B. Thomsen

Department of Hand Surgery, Skåne University Hospital Malmö, Malmö, Sweden machinery, and this pathway may be regulated differently in type 1 and type 2 diabetes; insulin, presence of extensive neuropathy, and/or other factors such as duration of diabetes and $\mathrm{HbA}_{1 \mathrm{c}}$ level may underlie this differential regulation.

Keywords Autophagy · Diabetes · Electron microscopy · Human $\cdot$ Neuropathy $\cdot$ Peripheral nerve

\section{Introduction}

Peripheral neuropathy, which affects more than half of all patients with diabetes $[1,2]$, is characterised by axonal atrophy, demyelination and a loss of nerve fibres that may result in loss of sensation, paraesthesia and pain. Hyperglycaemia, together with local ischaemia caused by microvascular disease, is believed to be one of the main factors underlying the development of diabetic peripheral neuropathy, but the exact cellular mechanisms involved are not known [3, 4]. Peripheral neuropathy in diabetes can also be a consequence of insulin-induced hypoglycaemic episodes [5-11], indicating that short periods of a low glucose level can be detrimental to peripheral nerves.

Autophagy (macroautophagy) is a degradation process responsible for clearance of the cell's damaged organelles and macromolecules. The autophagy pathway is initiated by the formation of membranes in the cytoplasm (phagophores) that subsequently internalise the damaged components and form double-membrane vesicles (autophagosomes). The degradation of these components occurs in the autolysosomes that form by fusion of autophagosomes with lysosomes $[12,13]$. By removal of damaged organelles and misfolded proteins, this pathway plays a critical role in cellular homeostasis and, therefore, dysfunction of this pathway is suggested to play a role in the development of various pathological conditions that affect the central nervous system, such as Alzheimer's 
disease and Parkinson's disease [14]. In peripheral nerves, signs of autophagy have been reported in nerve-growthfactor deprived sympathetic neurites in vitro [15], in transected rat sciatic axons [16], in sensory neurons in dorsal root ganglia of diabetic rats [17], as well as in sciatic nerves of a rat model with metabolic abnormalities [18]. Exposure of human neuroblastoma cells (SH-SY5Y) to sera from patients with diabetes and neuropathy increased the induction of autophagy compared with cells exposed to sera from healthy individuals or patients with diabetes but without neuropathy [19]. Furthermore, in insulin-induced hypoglycaemic rats, autophagy-related structures were observed in degenerating and regenerating axons and their associated Schwann cells [8]. Lack of autophagic activity in Schwann cells has been associated with neuropathic pain in a murine model of peripheral nerve lesion [20].

To our knowledge, no report has addressed the question of whether the autophagy pathway occurs in human peripheral nerves and whether this pathway is associated with neuropathy. In the current study, the aim was to search for signs of autophagy-related structures in the interosseous nerve of patients with or without diabetes, and to find out whether this pathway is associated with peripheral neuropathy in diabetes.

\section{Methods}

\section{Ethics}

The Ethical Review Board at Lund University approved the study protocol (LU 508-03). Informed consent was obtained from all participants. All qualitative and quantitative observations were performed on coded samples.

\section{Patients}

Patients with clinically and electrophysiologically verified carpal tunnel syndrome (CTS) were included. We excluded those with conditions associated with CTS other than diabetes [21]. Peripheral neuropathy was an exclusion criterion for patients with no diabetes, but not for patients with diabetes.

A biopsy of the posterior interosseous nerve that was not affected by pressure was taken while the patients underwent carpal tunnel release [21-23]. We randomly selected 23 patients from our previous study [21, 24], of which 14 had diabetes mellitus (seven type 1 and seven type 2) and nine control patients did not. The coded specimens were analysed by two of the authors who were not aware of the glucose status or presence of neuropathy among the patients.
Sample preparation

Nerve biopsies were fixed in cacodylate-buffered glutaraldehyde $(25 \mathrm{~g} / \mathrm{l})$ and osmium tetroxide $(10 \mathrm{~g} / \mathrm{l})$ as described previously [21]. The specimens were then dehydrated in a raising concentration of ethanol, infiltrated with propylene oxide and embedded in epoxy resin. Ultrathin sections $(60 \mathrm{~nm})$ were cut by Ultratome (Leica Microsystems, Wetzlar, Germany) and contrasted with $10 \%$ uranyl acetate for $20 \mathrm{~min}$ and $0.4 \%$ lead citrate for $3 \mathrm{~min}$. For qualitative description, the nerve specimens were studied directly in a JEOL JEM-1200EX transmission electron microscope (JEOL, Peabody, MA, USA).

\section{Electrophysiology}

Examinations were conducted with surface electrodes and skin temperature was kept above $30^{\circ} \mathrm{C}$ using a Viking Select Electromyograph (Viasys, Madison, WI, USA). The diagnosis of carpal tunnel syndrome was based on a fractionated measurement of antidromic sensory conduction velocity at the carpal tunnel segment $(<44 \mathrm{~m} / \mathrm{s})$ and distal motor latency for the median nerve ( $>4.1 \mathrm{~ms})$ [23].

To diagnose peripheral neuropathy, we measured sural nerve sensory conduction velocity, sural nerve antidromic action potential and peroneal nerve motor conduction velocity. Criterion for peripheral neuropathy was detection of abnormal values in both the sural and the peroneal nerves [25].

\section{Morphometry}

Electron micrographs were used for quantitative evaluations of the coded specimens, and ImageJ (http://imagej.nih.gov/ij/, version $1.47 \mathrm{q}$ ) was used for the analysis of data. The total number of myelinated nerve fibres was counted and their diameters (axon + myelin) were measured $(\times 1,000)$. The $g$ ratio values (axon diameter/fibre diameter) were calculated in $10 \%$ of myelinated axons for each sample. The density of myelinated nerve fibres (number of fibres $/ \mathrm{mm}^{2}$ ) was calculated by dividing the total number of fibres by the fascicular area. The area of the endoneurium was measured on micrographs taken from the whole nerve section.

For unmyelinated axons, the density was calculated by preparing 25 randomly selected electron micrographs (every third picture; $\times 15,000$ ), in each of which the number of fibres was counted. The area of the picture was measured and the density was calculated.

To evaluate the morphological signs of autophagy, the number and location of autophagy-related structures were evaluated on 25 randomly selected micrographs $(\times 15,000)$, using a modification of a previously used technique [26]. Dense osmophilic lysosomes, and structures resembling 
phagophores, autophagosomes and autolysosomes were included in the evaluation.

\section{Statistical analyses}

Data are presented as median (25th-75th percentiles) unless otherwise stated. Kruskal-Wallis rank sum tests followed by two-tailed Mann-Whitney $U$ tests were performed to analyse differences between the three groups. A $p$ value $<0.05$ was considered as statistically significant. Statistical analyses were performed using $\mathrm{R}$ statistical software (R Foundation for Statistical Computing, Vienna, Austria).

\section{Results}

\section{General observations}

The samples included one (16 samples), two (six samples) or three fascicles (one sample) enclosed by perineurium. Collagen fibres in the endoneurium occupied most of the space between the nerve fibres. Partial cleft in myelin at the Schmidt-Lanterman incisures were frequently observed. Unmyelinated axons showed normal morphology and had a mostly 1:1 relationship with the Schwann cells. Glycogen-like bodies were frequently observed in all type of cells in the endoneurium. Reich granules ( $\pi$ granules) and multilamellar zebra bodies were common, particularly in the Schwann cells of myelinated fibres and less frequently in fibroblasts and Schwann cells of unmyelinated axons (Fig. 1). In addition, osmophilic and osmophobic fat droplets were present in all type of cells. Autophagy-associated structures (i.e. phagophores, autophagosomes, electron-dense lysosomes and autolysosomes) were present in Schwann cells, axons, fibroblasts and endothelial cells (Fig. 2). The frequency of autophagy-associated structures varied between individuals, and was higher in nerves that showed extensive signs of pathology. The measurements demonstrated no difference in fascicle area between the different groups.
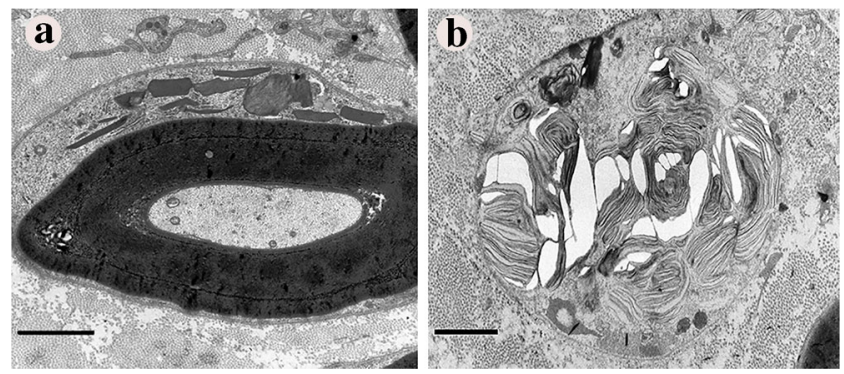

Fig. 1 Micrographs showing a Schwann cell including (a) Reich granules ( $\pi$ granules) and (b) zebra bodies. Scale bars, $2 \mu \mathrm{m}$
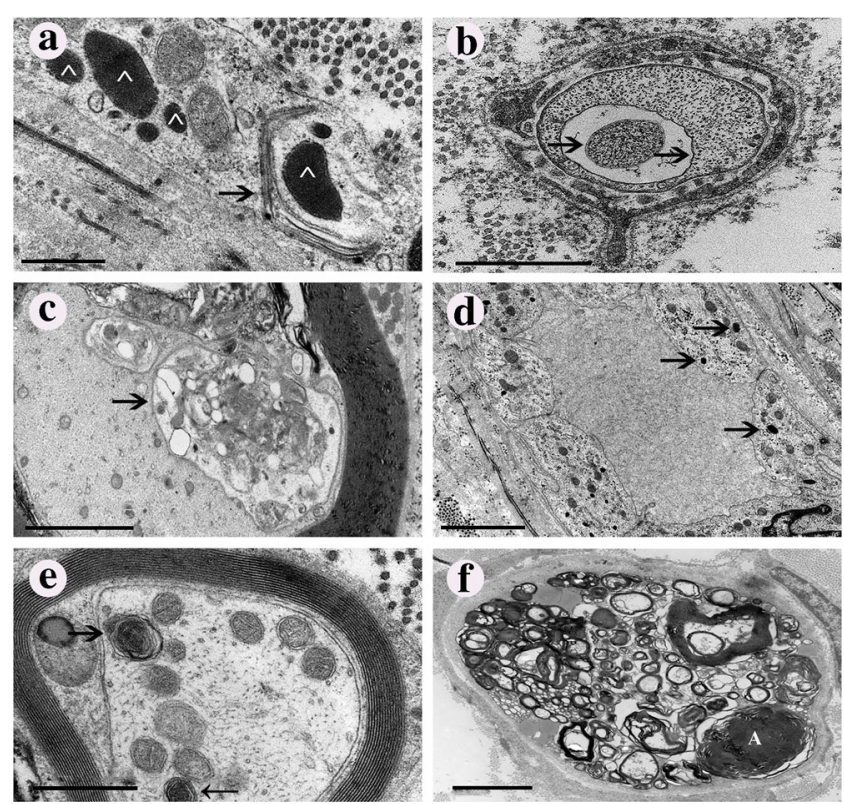

Fig. 2 Micrographs showing autophagy-associated structures in the human posterior interosseous nerve. (a) Phagophore (arrow) and electrondense lysosomes (arrowheads) in a fibroblast. (b, c) Autophagosomes in axons. Arrows in (b) show the double membrane of the autophagosomes, and the arrow in (c) shows an autophagosome. (d) Arrows show several electron-dense lysosomes in endothelial cells. (e) Autolysosomes in a myelinated axon are marked by arrows. (f) Autolysosomes (A) and myelin ovoids in a Schwann cell. Scale bars, $500 \mathrm{~nm}(\mathbf{a}, \mathbf{e}), 1 \mu \mathrm{m}$ (b) and $2 \mu \mathrm{m}(\mathbf{c}, \mathbf{d}, \mathbf{f})$

\section{Control patients}

The control group was composed of three men and six women with a median age of 51 years (range 38-69 years). The median $\mathrm{HbA}_{1 \mathrm{c}}$ level was 4.6\% (range 4.3-5.1\%); $27 \mathrm{mmol} / \mathrm{mol}$ (range $24-32 \mathrm{mmol} / \mathrm{mol}$ ).

Pathology and morphometry There was a small variation in morphology of the nerve samples in this group. While six samples showed normal morphology (Fig. 3a), three samples had some pathology in the form of a few myelin debris (1-4), demyelination of a few fibres ( $\leq 3)$, presence of remyelinated axons $(\leq 3)$, and late regenerated units including 3-11 myelinated axons.

The size distribution of myelinated fibres was bimodal and exhibited peaks at about 2-4 and 9-11 $\mu \mathrm{m}$ (Fig. 4a). The median fibre diameter was $7.3 \mu \mathrm{m}$ (Table 1), and $40 \%$ of the fibres had a diameter $\leq 5 \mu \mathrm{m}$. The g-ratio was 0.61 , and the median density for myelinated axons was $6,182 \mathrm{axons} / \mathrm{mm}^{2}$ (Table 1).

The median value for the diameter of unmyelinated axons was $0.9 \mu \mathrm{m}$; the size distribution of these fibres showed a unimodal pattern with a peak between $0.8-1.0 \mu \mathrm{m}$, and the median density of these fibres was 14,973 axons $/ \mathrm{mm}^{2}$ (Table 1). 


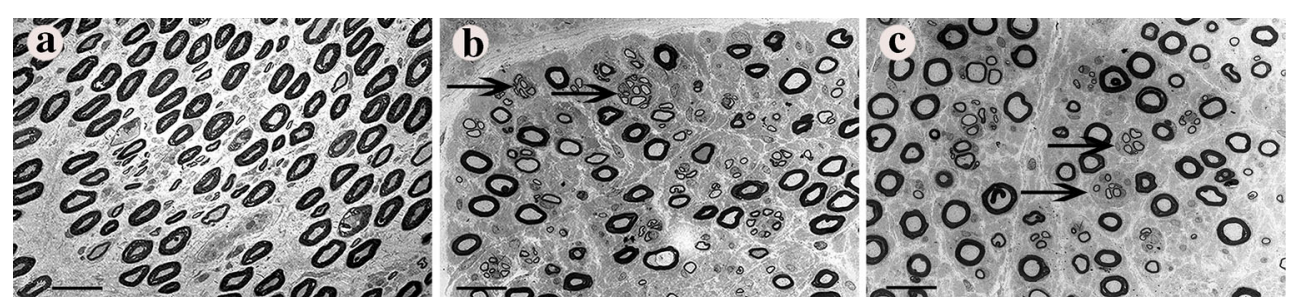

Fig. 3 Representative electron micrographs of the posterior interosseous nerve of patients who underwent carpal tunnel release. Samples were taken from (a) controls, who were patients without diabetes, (b) patients with type 2 diabetes and (c) patients with type 1 diabetes. Arrows show examples of regenerated units. Scale bars, $20 \mu \mathrm{m}$
The median density of autophagy-related structures was 4,286 per $\mathrm{mm}^{2}$ (Table 1), and $63.6 \%$ of these structures were electron-dense lysosomes. Almost 33\% of the autophagyrelated structures occurred in the Schwann cells.

Patients with type 2 diabetes

This group included four men and three women with a median age of 60 years (mean 55.6 years; range $41-67$ years). The median duration of the disease was 10 years (range 125 years), and the median $\mathrm{HbA}_{1 \mathrm{c}}$ level was $6.3 \%$ (range 5.2-9.0\%); $45 \mathrm{mmol} / \mathrm{mol}$ (range 33-75 $\mathrm{mmol} / \mathrm{mol}$ ), which was significantly higher than that in controls $(p=0.001)$.

Pathology and morphometry The presence of some small myelinated axons and a few late regenerated units indicated axonal degeneration and regeneration events in the past, and was taken as a sign of a mild neuropathy in four patients (Fig. 3b). The extensive neuropathy observed in three patients was indicated by widespread loss of large and medium-sized myelinated fibres, and presence of many small myelinated and unmyelinated axons. Collagen pockets, empty Schwann cells and cytoplasmic fat droplets were frequently observed in all samples. A sample from one patient (a 66-year-old man) included Renault bodies in the fascicles of the nerve.

The size distribution of myelinated nerve fibres was bimodal and exhibited peaks at about 2-3 and 9-10 $\mu \mathrm{m}$ (Fig. 4b). The myelinated nerve fibres were significantly smaller than those of controls $(p<0.0001$; Table 1$)$; as an example, $45 \%$ of fibres had a diameter $\leq 5 \mu \mathrm{m}$. The density of myelinated nerve fibres and the g-ratio were similar to the data obtained in the controls (Table 1).

The median diameter of unmyelinated axons was $0.8 \mu \mathrm{m}$, and the size distribution of these fibres showed a unimodal pattern with a peak at about $0.8-0.9 \mu \mathrm{m}$ that was close to the value obtained from the controls. The median density of unmyelinated axons was similar to that of the controls (Table 1).

The median density of autophagy-related structures was similar to that of the controls (Table 1), and $60 \%$ of these structures were electron-dense lysosomes. Almost $45 \%$ of the autophagy-related structures occurred in Schwann cells.

Patients with type 1 diabetes

This group included two men and five women with a median age of 48 years (range 39-58 years). The median duration of the disease was 28 years (range 12-43 years), and the median $\mathrm{HbA}_{1 \mathrm{c}}$ level was 7.4\% (range 6.9-9.9\%; $57 \mathrm{mmol} / \mathrm{mol}$ [range $50-85 \mathrm{mmol} / \mathrm{mol}])$, which was significantly higher than that in controls $(p<0.001)$ and patients with type 2 diabetes $(p<0.05)$.

Pathology and morphometry The nerve samples in these patients exhibited extensive signs of pathology as compared with samples from patients in other groups. Axonal degeneration, myelin debris, bands of Büngner, late regenerated units, and small-sized axons $(<3 \mu \mathrm{m})$ covered by thin myelin sheath were the hallmark of the pathology. Furthermore, the endoneurium in these samples contained many Schwann cells
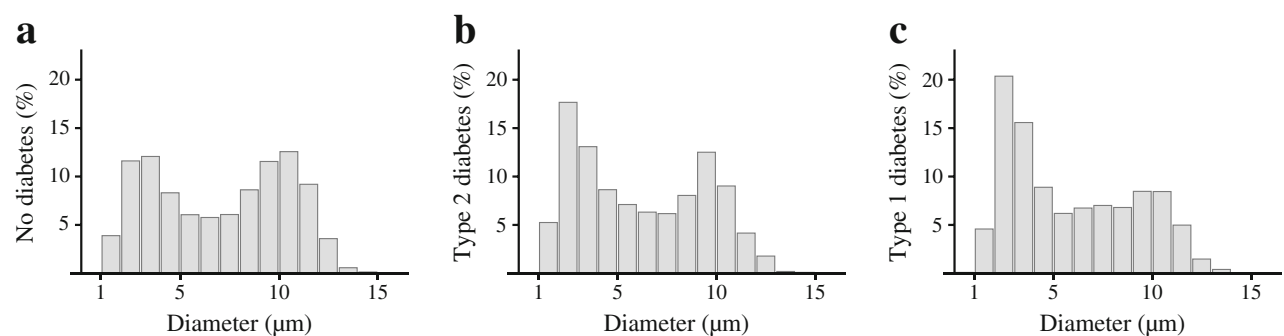

Fig. 4 The bar charts show the size distribution of myelinated fibres in the posterior interosseous nerve of patients who underwent carpal tunnel release. Patients with no sign of diabetes $(\mathbf{a} ; n=9)$, with type 2 diabetes $(\mathbf{b} ; n=7)$ or type 1 diabetes $(\mathbf{c} ; n=7)$ were included 
Table 1 Morphometric data from the posterior interosseous nerve of controls without diabetes and patients with type 1 and type 2 diabetes

\begin{tabular}{llll}
\hline Variable & $\begin{array}{l}\text { Controls } \\
(n=9)\end{array}$ & $\begin{array}{l}\text { Type 2 diabetes } \\
(n=7)\end{array}$ & $\begin{array}{l}\text { Type 1 diabetes } \\
(n=7)\end{array}$ \\
\hline Fascicle area $\left(\mu \mathrm{m}^{2}\right)$ & $123,480 \pm 16,074$ & $101,535 \pm 14,623$ & $108,269 \pm 14,080$ \\
Myelinated diameter $(\mu \mathrm{m})$ & $7.3(3.8-10.0)$ & $5.7(3.1-9.2)^{* * * *}$ & $5.0(3.0-8.8)^{* * * *, \dagger \dagger \dagger}$ \\
Unmyelinated diameter $(\mu \mathrm{m})$ & $0.9(0.7-1.0)$ & $0.8(0.6-1.0)$ & $0.8(0.6-1.0)^{* *} \dagger$ \\
Myelinated density $\left(\right.$ number $\left./ \mathrm{mm}^{2}\right)$ & $6,182(5,188-8,293)$ & $5,977(5,118-7,166)$ & $4,453(4,232-5,134)^{*}$ \\
Unmyelinated density $\left(\right.$ number $\left./ \mathrm{mm}^{2}\right)$ & $14,973(8,185-18,352)$ & $13,275(10,831-27,926)$ & $22,410(19,390-27,403)^{*}$ \\
g-ratio & $0.61(0.5-0.7)$ & $0.62(0.5-0.7)$ & $0.67(0.6-0.8)^{* * * *,+\dagger \dagger}$ \\
Autophagy structures $\left(\right.$ number $\left./ \mathrm{mm}^{2}\right)$ & $4,286(3,571-5,357)$ & $3,486(1,943-4,862)$ & $10,925(6,147-13,123)^{\dagger \dagger}$ \\
\hline
\end{tabular}

Values are presented as median (25th-75th percentiles)

Fascicle area is presented as means \pm SEM

${ }^{*} p<0.05,{ }^{* *} p<0.01,{ }^{* * *} p<0.0001$ vs control; ${ }^{\dagger} p<0.05,{ }^{\dagger \dagger} p<0.01,{ }^{\dagger \dagger} p<0.001,{ }^{\dagger \dagger \dagger} p<0.0001$ vs type 2 diabetes

without any associated axons. Macrophages were frequently observed in the endoneurium, and osmophilic and osmophobic fat droplets were often located in the Schwann cells, fibroblasts and endothelial cells. Aggresomes were common in the axons (Fig. 5a) and Schwann cell cytoplasm. A sample from one patient (a 46-year-old women) included several Renault bodies in both fascicles of the nerve. These bodies occupied almost half of the endoneurium (Fig. 5b).

The size distribution of myelinated nerve fibres was bimodal and exhibited peaks at about 2-3 and 9-11 $\mu \mathrm{m}$ (Fig. 4c). The median fibre diameter was significantly smaller in this group than in controls $(p<0.0001)$ or patients with type 2 diabetes $(p<0.0001$; Table 1$)$; approximately $50 \%$ of fibres had a diameter $\leq 5 \mu \mathrm{m}$. In this group, g-ratios were significantly higher than those obtained in controls $(p<0.0001)$ or patients with type 2 diabetes $(p<0.001)$, and the density of these fibres was significantly lower than that of controls $(p<0.05$; Table 1).

The median diameter of unmyelinated axons was $0.8 \mu \mathrm{m}$ (Table 1), and the size distribution of these fibres showed a unimodal pattern with a peak between 0.6 and $0.8 \mu \mathrm{m}$ that was significantly smaller than that in controls $(p<0.01)$ or patients with type 2 diabetes $(p<0.05)$. The density of unmyelinated axons was significantly higher in these patients than in patients without diabetes $(p<0.05)$.

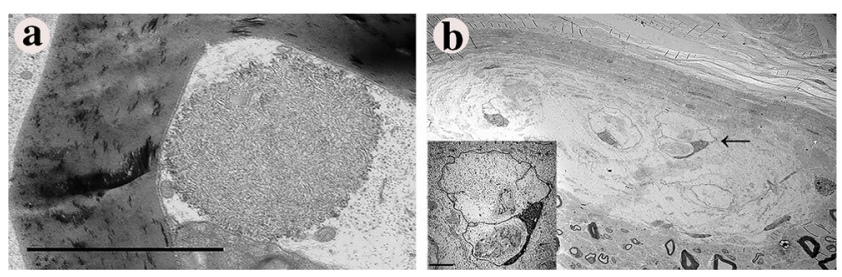

Fig. 5 (a) Aggresomes were common in the axons of patients with type 1 diabetes. (b) A sample from one of these patients (a 46-year-old woman) included several Renault bodies (arrow) in two fascicles of the nerve. These bodies occupied almost half of the endoneurium. Scale bar is $2 \mu \mathrm{m}$ (a) and $5 \mu \mathrm{m}$ (b; inset)
The median density of autophagy-related structures in these samples was significantly higher than that in patients with type 2 diabetes $(313 \%, p<0.01$; Table 1$)$. The median density of autophagy-related structures was also higher in patients with type 1 diabetes than controls, but the difference was not significant $(p=0.06)$. Of these structures, $77.8 \%$ were electron-dense lysosomes, and $25.8 \%$ of the autophagyrelated structures occurred in Schwann cells.

\section{Discussion}

Dysfunction of the autophagy pathway is reported in many pathological conditions in the central nervous system, but autophagy in the peripheral nervous system has not received much attention. In the current study, we addressed the question of whether the autophagy pathway occurs in human peripheral nerves and whether this pathway is associated with neuropathy. We report here that ultrastructural signs of the autophagy pathway occur in the uncompressed posterior interosseous nerve of the forearm in individuals with and without diabetes, which suggests that peripheral nerves have access to the autophagy machinery for turnover of damaged cellular compartments. Our main finding was that the number of autophagy-associated structures was significantly higher in the nerves of patients with type 1 than type 2 diabetes. The frequency of these structures in type 1 diabetes was also higher than in controls, but the difference was not significant $(p=0.06)$. The pattern of neuropathy was similar in both groups of patients with diabetes, showing axonal degeneration and regeneration, although patients with type 1 diabetes showed distinct and extensive signs of pathology. The picture in patients with type 2 diabetes was diverse, from being almost normal to being extensively abnormal (discussed later). Hence, the high number of autophagy-related structures in patients with type 1 diabetes may be related to the presence of neuropathy in all patients in this group. Interestingly, 
Towns and colleagues observed induced autophagy in human neuroblastoma cells that were exposed to sera from patients with diabetes and neuropathy, but not from patients with diabetes without neuropathy [19]. Together, these data suggest that there is a relationship between the presence of neuropathy in the peripheral nerve and the autophagy pathway.

The increased number of autophagy-related structures in samples from patients with type 1 diabetes raises an important question, i.e. whether autophagy is regulated differently in type 1 and type 2 diabetes. Differences might include increased production of autophagy-related structures, or a delayed or blocked autophagy pathway leading to the accumulation of these structures in samples from patients with type 1 diabetes. Dysfunction of the insulin-signalling pathway has been suggested to be involved in autophagy-mediated clearance of aggregated proteins in an in vitro model of Huntington's disease [27]. Recently, Lv and colleagues [28] observed that autophagy was inhibited in glucose-infused hyperglycaemic muscle in rats, but it was enhanced in the muscle of streptozotocin-induced diabetes-related hyperglycaemia, suggesting a role for insulin in the regulation of autophagy. Insulin is not involved in glucose uptake by neurons; however, as a growth factor, it plays an essential role in development and survival of neurons. In type 2 diabetes, neurons can develop insulin resistance, which makes them vulnerable to metabolic disturbances $[29,30]$. This insulin resistance is suggested to be one of the reasons why enhanced glycaemic control has only a limited effect on prevention of neuropathy in type 2 diabetes, whereas the effect in type 1 diabetes is more substantial $[29,30]$. The autophagy pathway, from the formation of phagophores and autophagosomes to the fusion of autophagosomes with lysosomes, involves autophagy-related genes and proteins. The autophagy-related proteins are, in turn, regulated by the mTOR (mammalian target of rapamycin) kinase pathway, and the activity of this pathway is controlled by insulin and other growth factors [31]. If the insulin-signalling pathway has a significant impact on the autophagy pathway, then it is possible that autophagy is regulated differently in type 1 and type 2 diabetes. We cannot, however, exclude the possibility that factors other than insulin may underlie the high density of autophagyrelated structures in the nerves of patients with type 1 diabetes in the current study.

The question is what the biological significance of the autophagy pathway is in the peripheral nerve. Kosacka and colleagues have recently reported the presence of autophagyrelated proteins and structures in the sciatic nerve of a rat model with insulin resistance and other metabolic disturbances [18]. The authors, however, did not record any electrophysiological or morphological signs of neuropathy and they, therefore, suggest that autophagy may have protected these rats against development of neuropathy. We have previously shown that signs of autophagy in peripheral nerves of hypoglycaemic rats occur mainly in association with regeneration of axons [8], suggesting that autophagy may have a role in survival of the axons. Further investigation is needed to determine whether autophagy has a protective role in the peripheral nerve.

In the study performed by Mohseni, signs of autophagy were not present in healthy control rats [8]. In the current study of human peripheral nerves, we frequently observed autophagy-related structures, particularly electron-dense lysosomes, in the control individuals. In addition, nearly all of the nerves taken from these patients included a few degenerated/ regenerated axons. Hence, it is possible that the presence of autophagy-related structures in the human peripheral nerves is a normal age-related condition [32]; however, it is also possible that the frequency of these structures in mild neuropathy is raised even above the normal aged-related level.

For qualitative and quantitative analysis of autophagy, we used transmission electron microscopy, which is considered a useful and important tool $[12,26]$. For quantification, we focused on the following structures: phagophores and autophagosomes, autolysosomes and electron-dense lysosomes. Isolated phagophores have a double membrane and autophagosomes are double-membrane vacuoles; however, not all double-membrane structures are autophagy-related. The rough endoplasmic reticulum, for example, shows a similar structure to autophagophores, and the presence of ribosomes can be a helpful criterion to distinguish them from autophagy-related structures. Furthermore, in the axons, the invagination of the axolemma can appear as lucid doublemembrane vacuoles. In addition, damaged mitochondria most often have disturbed cisterna and show a morphology similar to autophagosomes. In order to avoid misinterpretation, we included only those double-membrane vacuoles that contained cytoplasm and/or intact organelles in our evaluation. Autolysosomes usually have a single membrane and include debris of cytoplasmic content and could, therefore, easily be recognised in our samples. We also quantified electron-dense lysosomes in the cells. Transmission electron microscopy is a highly valuable method for the recognition of autophagy structures, but to avoid bias it is desirable to supplement the analysis with other methods, such as immunohistochemistry, to detect phagophore/autophagosome-related proteins, such as microtubule-associated protein 1A/1B-light chain 3. Use of immunohistochemistry was, however, not an option in the current study due to the lack of access to tissue with appropriate fixation for the technique. Despite this limitation, our results strongly suggest that the human peripheral nerve has access to the autophagy machinery.

Regarding neuropathy, the results of the current study show that axonal degeneration and regeneration occurs in patients with type 1 and type 2 diabetes. Absence of large $(>2 \mu \mathrm{m})$ unmyelinated axons suggests that primary demyelination was not a part of the pathology in the patients with diabetes. 
Samples from patients with type 2 diabetes showed a large variation in respect to neuropathy; samples from three patients showed extensive loss of myelinated and unmyelinated axons, and those taken from another four patients showed a picture similar to that observed in control individuals. Nerves taken from patients with type 1 diabetes, however, showed a more uniform pattern with degeneration of large myelinated axons. Higher density of small unmyelinated axons in samples from patients with type 1 diabetes vs type 2 diabetes suggests a higher rate of axonal regeneration in the former group, which confirms results reported in other publications $[33,34]$.

\section{Conclusions}

Owing to ethical considerations, it is difficult to obtain human nerve samples for morphological investigation. The majority of knowledge is therefore based on experimental studies. We acknowledge that the small number of patients in the current study limits our ability to detect significant differences between groups, and interpretation of our results should be conducted with caution. With this limitation in mind, our results show that autophagy-related structures are present in the human peripheral nerve, and that autophagy may be regulated differently in type 1 vs type 2 diabetes. It is possible that insulin, presence of extensive neuropathy, and/or other factors such as duration of diabetes and $\mathrm{HbA}_{1 \mathrm{c}}$ level underlie the extensive occurrence of autophagy in type 1 diabetes. The most important question is whether the autophagy pathway is the cause or the consequence of neuropathy in diabetes. Further experiments are needed to answer this question.

Acknowledgements We thank M. Bagheri (Linköping University, Sweden) for help with preparation of the microscopic pictures for publication.

Funding This work was supported by the Swedish Research Council (Medicine), Svenska Diabetesförbundet, Diabetesföreningen Malmö, Stiftelsen Sigurd och Elsa Goljes Minne, Linköping University, Lund University, Region Skåne, Funds from the Skåne University Hospital Malmö, Sweden, and Ministry of Agriculture and Animal Resources, Red Sea State, Sudan.

Duality of interest The authors declare that there is no duality of interest associated with this manuscript.

Contribution statement LBD and NOBT were responsible for inclusion and pre- and postoperative characterisation of the patients as well as performing the nerve biopsies in all patients, and the random selection of the present samples from the original publication. AAMO and SM performed sample sectioning, staining and electron microscopy. SM was responsible for qualitative description of autophagy and neuropathy, and AAMO was responsible for quantification of neuropathy. All authors were involved in analysis and interpretation of data, and were involved in drafting the manuscript and approval of the final version. SM is responsible for the integrity of the work as a whole.

\section{References}

1. Dyck P, Kratz K, Karnes J et al (1993) The prevalence by staged severity of various types of diabetic neuropathy, retinopathy, and nephropathy in a population-based cohort: the Rochester Diabetic Neuropathy Study. Neurology 43:817-824

2. Edwards J, Vincent A, Cheng H, Feldman E (2008) Diabetic neuropathy: mechanisms to management. Pharmacol Ther 120:1-34

3. Low PA, Nickander KK, Tritschler HJ (1997) The roles of oxidative stress and antioxidant treatment in experimental diabetic neuropathy. Diabetes 46(Suppl 2):S38-S42

4. Sheetz MJ, King GL (2002) Molecular understanding of hyperglycemia's adverse effects for diabetic complications. JAMA 288:2579-2588

5. Jamali R, Mohseni S (2005) Hypoglycaemia causes degeneration of large myelinated nerve fibres in the vagus nerve of insulin-treated diabetic BB/Wor rats. Acta Neuropathol 109:198-206

6. Jamali R, Mohseni S (2006) Differential neuropathies in hyperglycemic and hypoglycemic diabetic rats. J Neuropathol Exp Neurol 65: $1118-1125$

7. Mohseni S (2001) Hypoglycemic neuropathy. Acta Neuropathol 102: 413-421

8. Mohseni S (2011) Autophagy in insulin-induced hypoglycaemic neuropathy. Pathology 43:254-260

9. Mohseni S, Hildebrand C (1998) Hypoglycaemic neuropathy in BB/ Wor rats treated with insulin implants: electron microscopic observations. Acta Neuropathol 96:151-156

10. Mohseni S, Hildebrand C (1998) Neuropathy in diabetic BB/Wor rats treated with insulin implants. Acta Neuropathol 96:144-150

11. Mohseni S, Lillesaar C, Hildebrand C, Theodorsson E (2000) Hypoglycaemic neuropathy: occurrence of axon terminals in plantar skin and plantar muscle of diabetic BB/Wor rats treated with insulin implants. Acta Neuropathol 99:257-262

12. Klionsky DJ, Abdalla FC, Abeliovich $\mathrm{H}$ et al (2012) Guidelines for the use and interpretation of assays for monitoring autophagy. Autophagy 8:445-544

13. Sridhar S, Botbol Y, Macian F, Cuervo AM (2012) Autophagy and disease: always two sides to a problem. J Pathol 226:255-273

14. Wong E, Cuervo AM (2010) Autophagy gone awry in neurodegenerative diseases. Nat Neurosci 13:805-811

15. Yu L-Y, Jokitalo E, Sun Y-F et al (2003) GDNF-deprived sympathetic neurons die via a novel nonmitochondrial pathway. J Cell Biol 163: 987-997

16. Piao Z, Wang W, Xu X et al (2004) Autophagy of neuron axon during regeneration of rat sciatic nerves. J First Mil Med Uni 2: 361-364

17. Gonzalez CD, Lee M-S, Marchetti P et al (2011) The emerging role of autophagy in the pathophysiology of diabetes mellitus. Autophagy 7:2-11

18. Kosacka J, Nowicki M, Blüher M et al (2013) Increased autophagy in peripheral nerves may protect Wistar Ottawa Karlsburg W rats against neuropathy. Exp Neurol 250:125-135

19. Towns R, Kabeya Y, Yoshimori T et al (2005) Sera from patients with type 2 diabetes and neuropathy induce autophagy and colocalization with mitochondria in SY5Y cells. Autophagy 1:163-170

20. Marinelli S, Nazio F, Tinari A et al (2014) Schwann cell autophagy counteracts the onset and chronification of neuropathic pain. Pain 155:93-107

21. Thomsen NOB, Mojaddidi M, Malik RA, Dahlin LB (2009) Reduced myelinated nerve fibre and endoneurial capillary densities in the forearm of diabetic and non-diabetic patients with carpal tunnel syndrome. Acta Neuropathol 118:785-791

22. Thomsen NOB, Mojaddidi M, Malik RA, Dahlin LB (2009) Biopsy of the posterior interosseous nerve: a low morbidity method for assessment of peripheral nerve disorders. Diabet Med 26:100-104 
23. Thomsen NO, Cederlund R, Rosen I, Björk J, Dahlin LB (2009) Clinical outcomes of surgical release among diabetic patients with carpal tunnel syndrome: prospective follow-up with matched controls. J Hand Surg [Am] 34:1177-1187

24. Mojaddidi M, Ahmed M, Ali R et al (2014) Molecular and pathological studies in the posterior interosseous nerve of diabetic and non-diabetic patients with carpal tunnel syndrome. Diabetologia 57:1711-1719

25. England JD, Gronseth GS, Franklin G et al (2005) Distal symmetric polyneuropathy: a definition for clinical research: report of the American Academy of Neurology, the American Association of Electrodiagnostic Medicine, and the American Academy of Physical Medicine and Rehabilitation. Neurology 64:199-207

26. Backues SK, Chen D, Ruan J, Xie Z, Klionsky DJ (2014) Estimating the size and number of autophagic bodies by electron microscopy. Autophagy 10:155-164

27. Yamamoto A, Cremona ML, Rothman JE (2006) Autophagymediated clearance of huntingtin aggregates triggered by the insulin-signaling pathway. J Cell Biol 172:719-731
28. Lv P, Huang J, Yang J et al (2014) Autophagy in muscle of glucoseinfusion hyperglycemia rats and streptozotocin-induced hyperglycemia rats via selective activation of m-TOR or FoxO3. PLoS One 9: e87254

29. Callaghan B, Feldman E (2013) The metabolic syndrome and neuropathy: therapeutic challenges and opportunities. Ann Neurol 74:397-403

30. Callaghan BC, Hur J, Feldman EL (2012) Diabetic neuropathy: one disease or two? Curr Opin Neurol 25:536-541

31. Nixon RA (2006) Autophagy in neurodegenerative disease: friend, foe or turncoat? Trends Neurosci 29:528-535

32. Yue Z, Friedman L, Komatsu M, Tanaka K (2009) The cellular pathways of neuronal autophagy and their implication in neurodegenerative diseases. Biochim Biophys Acta 1793:1496-1507

33. Malik RA (1997) The pathology of human diabetic neuropathy. Diabetes 46(Suppl 2):S50-S53

34. Malik RA, Veves A, Walker D et al (2001) Sural nerve fibre pathology in diabetic patients with mild neuropathy: relationship to pain, quantitative sensory testing and peripheral nerve electrophysiology. Acta Neuropathol 47:367-374 